\title{
Investigation into Methods for Predicting Connection Temperatures
}

K. Anderson, M. Gillie

The mechanical response of connections in fire is largely based on material strength degradation and the interactions between the various components of the connection. In order to predict connection performance in fire, temperature profiles must initially be established in order to evaluate the material strength degradation over time.

This paper examines two current methods for predicting connection temperatures: The percentage method, where connection temperatures are calculated as a percentage of the adjacent beam lower-flange, mid-span temperatures; and the lumped capacitance method, based on the lumped mass of the connection. Results from the percentage method do not correlate well with experimental results, whereas the lumped capacitance method shows much better agreement with average connection temperatures.

A $3 D$ finite element heat transfer model was also created in Abaqus, and showed good correlation with experimental results.

Keywords: Connection, joint, heat transfer, steel, concrete, fire, temperature.

\section{Introduction}

Structural fire design is, to a large extent, based on single member tests. Due to the nature of these tests, the behaviour of the connections is neglected suggesting that they do not play a critical role in fire. In support of this theory, connections generally have a lower temperature than the surrounding structure during fires and are usually protected. This assumption of cooler connections is valid but this does not justify ignoring them in fire design. During both heating and cooling, connections will be subject to conditions, for example large moments and shear forces, which they will not typically have been designed for [1]. The response of connections to these conditions is complex and is largely based on the material strength degradation and the interactions between the various components of the connection. To predict how the behaviour of connections affects global performance in fire, temperature profiles must initially be established in order to evaluate the material strength degradation over time.

This paper examines two current methods available for predicting connection temperatures as defined in Eurocode 3 [2]. The first of these methods suggests that connection temperatures can be defined as a percentage of the adjacent beam temperature, and a second is based on the lumped mass of material at the connection. A 3D finite element model is also created to predict connection temperatures using the commercial software package Abaqus [3]. Abaqus uses heat transfer theory to predict connection temperatures over time. These methods are all compared to experimental data and the validity and accuracy of each is evaluated and its limitations explored.

\section{Theory}

\subsection{Eurocode percentages method}

Eurocode 3 details two methods for predicting connection temperatures. The first assumes that connection temperatures follow the same general trend as local beam temperatures but are a percentage lower. This method simplifies connections into 2 categories based on whether the connect- ing beam is less than or equal to $400 \mathrm{~mm}$ deep, or greater than that. In the first case, where the top of the connection is adjacent to the concrete slab, connection temperatures are approximated at $88 \%$ of the beam lower flange mid-span temperature at the bottom of the connection, $75 \%$ at mid-height and $62 \%$ at the top. Between these points the temperature is assumed to vary linearly. Where the connecting beam is more than $400 \mathrm{~mm}$ deep, temperatures are calculated as $88 \%$ of the beam temperature at the bottom and mid-height of the connection, tapering to $70 \%$ at the top.

Connections are cooler at the top for two main reasons. The largest contributor to heating is radiation from hot surfaces such as compartment or furnace walls. Where there is no direct line of sight between these walls and the member, in this case the connection, the radiative heating will decrease. This phenomenon is known as shadowing and causes the top of the connection to be cooler than the rest. The provision of a heat sink in the form of a concrete slab will also reduce connection temperatures.

\subsection{Lumped capacitance}

Connection temperatures can also be predicted using the ratio of material volume to exposed surface area. An average connection temperature or that of a specific connection component, such as a bolt or end plate, can be calculated, assuming the gas temperature-time curve is known.

The lumped capacitance method [4] calculates the uniform temperature rise in an unprotected steel member using a series of finite time steps $\Delta t$, as given in Eq. (1).

$$
\Delta T_{\mathrm{s}}=\frac{h}{C_{\mathrm{s}} \frac{W}{D}}\left(T_{\mathrm{f}}-T_{\mathrm{s}}\right) \Delta t,
$$

where $h$ heat transfer coefficient,

$T_{\mathrm{f}}$ gas temperature,

$T_{\mathrm{s}} \quad$ steel temperature,

$C_{\mathrm{s}} \quad$ steel specific heat,

$D \quad$ heated perimeter,

$W \quad$ steel volume per meter length. 
When applied to connections, this method must take into consideration the large volume of steel at the connection. Therefore the equation has been modified to use the ratio of volume of steel, $V$, to heated surface area, $A$, as shown in Eq. (2).

$$
\Delta T_{\mathrm{s}}=\frac{h}{C_{\mathrm{s}} \frac{V}{A}}\left(T_{\mathrm{f}}-T_{\mathrm{s}}\right) \Delta t .
$$

This method does not account for the concrete slab above the connection and, as the effect of the slab on connection temperatures has not been well researched, the validity of applying this method to connections is uncertain.

\subsection{Finite Element Modelling}

An alternative means of predicting structural temperatures is to carry out a finite-element heat transfer analysis. Detailed temperature profiles can be created and this information can be used as direct input for a structural model. In principle, this method is highly accurate, however obtaining correct values for the all input parameters is very challenging. The modelling process is outlined below.

For the purpose of this paper, the gas temperature-time curve to which a connection is exposed is assumed to be known, therefore the modelling will be limited to the convective and radiative heat transfer between the gas and solid, and to the conduction between the connection components. Perfect conduction is assumed between the various connection components such as bolts and bolt holes and through the welds.

Convective heat transfer is heating by movement of the hot gases, where the heat flux due to convection, $q_{\mathrm{c}}^{\prime \prime}$ and is given by:

$$
q_{\mathrm{c}}^{\prime \prime}=h\left(T_{\mathrm{f}}-T_{\mathrm{su}}\right),
$$

where $T_{\text {su }}$ is the surface temperature.

The heat transfer coefficient varies with temperature and depends on the hot gas velocity. Its value for structural steel is given in Eurocode 3 as $25 \mathrm{~W} / \mathrm{m}^{2} \cdot \mathrm{K}$ [2]. At the connections, the convective heating will be less, due to lower gas velocities in these areas. However, no practical methods exist for accurately calculating heat transfer coefficient at connections. This paper has therefore used the Eurocode recommended value. This assumption was verified with a sensitivity study.

Radiative heat transfer is heating directly between one item and another or between the fire source and a structural element or between one structural element and other. Emissivity is used to describe the radiative power of an object and can be defined as the ratio of the radiative power of the object to the radiative power of a black body where a black body is a perfect emitter and emissivity can never be greater than 1 .

Emissivity varies with temperature and in large building fires is usually the dominant mode of heating. There are a huge number of factors which affect radiative heating, for example the make up of the air in the room: if the air contains soot particles the radiation between objects will be lower than in clear air, or if an element becomes charred or sooty its emissive power will reduce, i.e. less heat will be absorbed by the element. Due to the many variables affecting emissivity, predicting radiative heating is extremely difficult: for one structural member, there will be variations not only with tem- perature but also with factors such as location in the building, fuel type and ventilation conditions.

The heat flux due to radiative heating, or total emissive power of an object is given in Eq. (4).

$$
q_{\mathrm{e}}^{\prime \prime}=\varepsilon \sigma T_{\mathrm{su}}^{4},
$$

where $\varepsilon \quad$ emissivity and

\section{$\sigma \quad$ Stefan Boltzmann constant.}

The emissivity at a connection will be lower than that of the local beams and columns due to the shadow effect, as discussed in section 2.1. Eurocode 3 suggests that for 'shadowed' areas a reduction factor for unprotected steel temperatures can be defined as shown in Eq. (5).

$$
k_{\mathrm{sh}}=\frac{\left[\frac{A_{\mathrm{m}}}{V}\right]_{\mathrm{b}}}{\left[\frac{A_{\mathrm{m}}}{V}\right]},
$$

$$
\begin{aligned}
& \text { where }\left[\frac{A_{\mathrm{m}}}{V}\right]_{\mathrm{b}} \quad \text { box section factor, } \\
& {\left[\frac{A_{\mathrm{m}}}{V}\right] \quad \text { section factor, }} \\
& A_{\mathrm{m}} \quad \text { surface area of the member per unit length. }
\end{aligned}
$$

This method is suggested for beams but has not yet been validated for connections. Further research is required to validate this assumption.

\section{Results}

Two sets of experimental data have been used to investigate the accuracy of these methods for predicting connection temperatures. These are briefly summarised here.

- Manchester University furnace tests carried out in 2008 [5-6] consisting of 4 beams spanning from one column with a concrete slab on top. The steel members were all unprotected. This whole assembly was tested in a furnace where the gas temperatures followed a 60 minute standard fire. Connection temperatures were recorded at several locations. Cooling was not considered. 4 connection types were used: flush and flexible end plate, fin plate and web cleats. The flush end plate and fin plate have been used for validation in this paper.

- Cardington full scale tests from January 2003 [7]. This was a compartment fire test on the $4^{\text {th }}$ storey of an 8 storey building where one of the main objectives was to monitor the connection behaviour including temperature evolution during the heating and cooling phases. The interior beam-to-column connections were flexible end plates. The columns were protected to the underside of the beams whilst the connection remained unprotected.

\subsection{Eurocode Percentages Method}

The Eurocode percentages method was used to predict the temperatures of two connections, a flush end plate from the Manchester University tests and a flexible end plate from the Cardington tests. The results of these are shown in Figs. 1 and 2.

Fig. 1 shows the predicted temperatures at 3 locations on the connection, bottom, mid-height and top, in comparison 


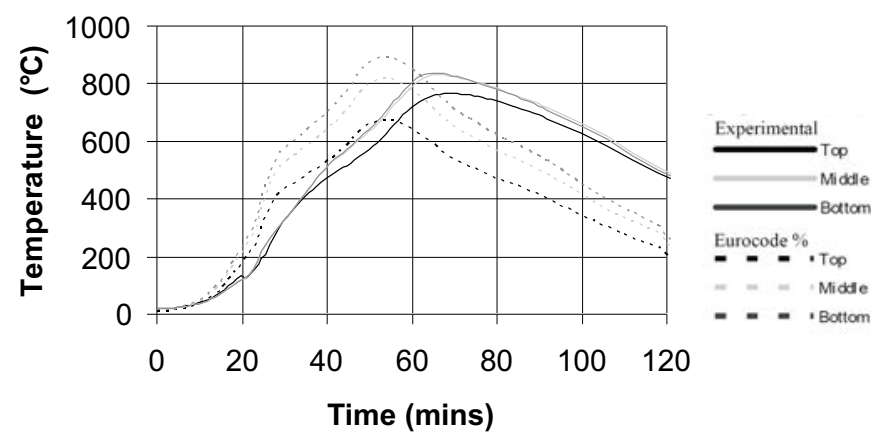

Fig. 1: EC \% method: flexible end plate

to the recorded temperatures at the same locations. For the first 15 minutes the predicted temperatures are of reasonable accuracy but after this point predicted temperatures are much higher than experimental temperatures. After 50 minutes the predicted temperature of the bottom of the connection is $900{ }^{\circ} \mathrm{C}$, whereas the measured temperature was $250{ }^{\circ} \mathrm{C}$ lower at $650{ }^{\circ} \mathrm{C}$; there is a similar error margin for the mid-height temperature. The peak temperature of the connection is estimated to be at around 50 minutes, coinciding with the peak beam temperature. Connections, however, can continue to heat after the surrounding structure has started cooling and experimental results show that this connection does not start cooling until 15 minutes later. During the cooling stage the connection temperatures are underpredicted by between $150{ }^{\circ} \mathrm{C}$ and $250{ }^{\circ} \mathrm{C}$ for the 60 minute cooling period at all locations.

The results for the flush end plate, Fig. 2, show that the temperatures at three locations on the connection are initially over-conservative by up to $200{ }^{\circ} \mathrm{C}$ but are equally under-conservative after about 25 minutes until the conclusion of the test. The trend of connection temperatures relative to one another is also not shown: The experimental test shows connection temperatures varying by around $75 \mathrm{EC}$ from top to bottom, whereas the Eurocode method shows a variation of close to $200^{\circ} \mathrm{C}$.

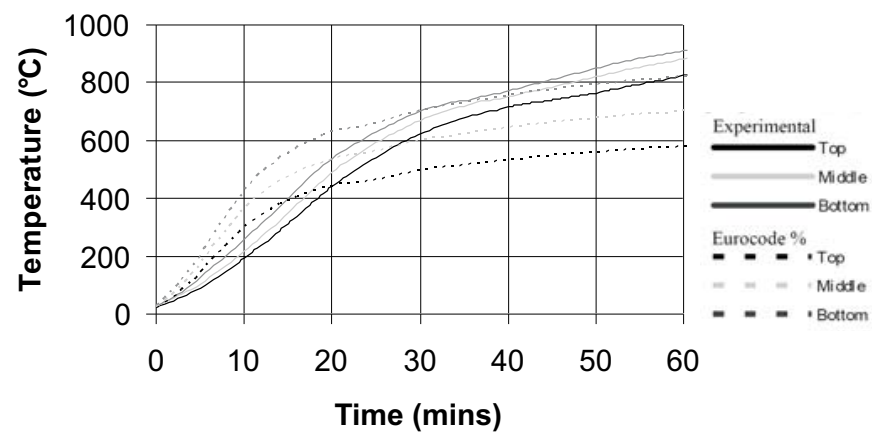

Fig. 2: EC \% method: flush end plate

This method provides a very simple means of estimating connection temperatures where the only information required is the beam mid-span temperature and depth. However, results show it to be unreliable in both heating and, to a larger extent, in cooling. The implication of this is that the use of this method is inappropriate except for very crude calculations.

\subsection{Lumped capacitance}

The lumped capacitance method has been used to predict the average temperature of a fin-plate connection from the Manchester University tests and of the flexible end plate used in the Cardington tests. The results are shown in Fig. 3 and compared to the recorded average connection temperature.

For fin-plate, the average temperature is predicted well. However it is noteworthy that this experiment was carried out in a highly controlled environment. The same lumped capacitance method is then used to calculate the average temperature for the flexible end plate. The temperatures predicted are consistently higher than the experimental results by between $30{ }^{\circ} \mathrm{C}$ and $90{ }^{\circ} \mathrm{C}$ and are therefore conservative. However, there is a good correlation between the predicted and experimental trend.

More input data is required for this method than for the percentages method: connection geometry and gas temperature-time curve. Despite calculations being basic, results show that good average temperatures are predicted. There are, however, many factors that could affect the results, such as how much of the beam or column is considered to be part of the connection and what effect the concrete slab has on the heating rate. As the effect of the slab on connection temperatures has not been well researched, this assumption may be invalid. Also, temperature gradients are present over connections, and mechanical response may vary notably between a connection with one average temperature to that with a temperature profile. Therefore an average temperature may not be adequate for detailed calculation purposes.

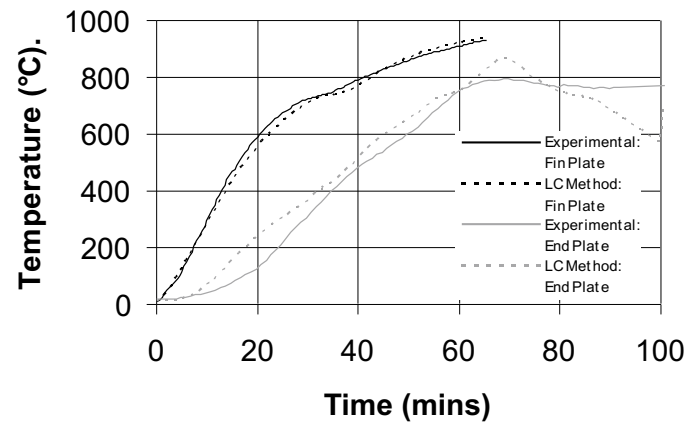

Fig. 3: Lumped capacitance method for fin plate and flexible end plate

\subsection{Finite Element Modelling}

A model of the flexible end plate was created in Abaqus for the 200 minute fire. It includes a 140 minute cooling phase. In creating the finite element model there were three main areas for consideration: radiative heating, convective heating and the inclusion of a concrete slab.

A sensitivity study was carried out to look at these three parameters and examine their effect on results. It was found that varying the heat transfer coefficient, and therefore the level of convective heating, at the connection had a negligible effect on the results. When the concrete slab was included in the model the temperatures of the upper flanges of the beams were affected, but other temperature predictions remained unchanged. Based on these results the concrete slab was excluded from further modelling. 
The value of emissivity affected the results, and therefore the area near to the connection was assigned a lower emissivity than the rest of the structure. This is based on the shadow effect in this location, as discussed in section 2.1.

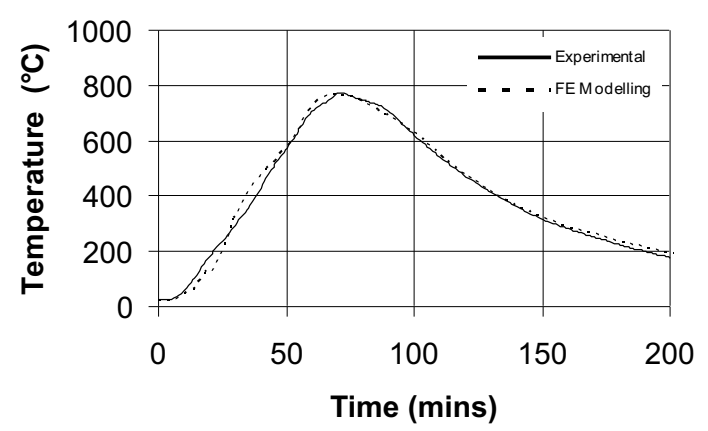

Fig. 4. Finite element for flexible end plate: top of connection

The known gas temperature over time was input to the model and heating assumed on all faces apart from the upper flanges of the beams and the top of the column where there was contact with the concrete slab.

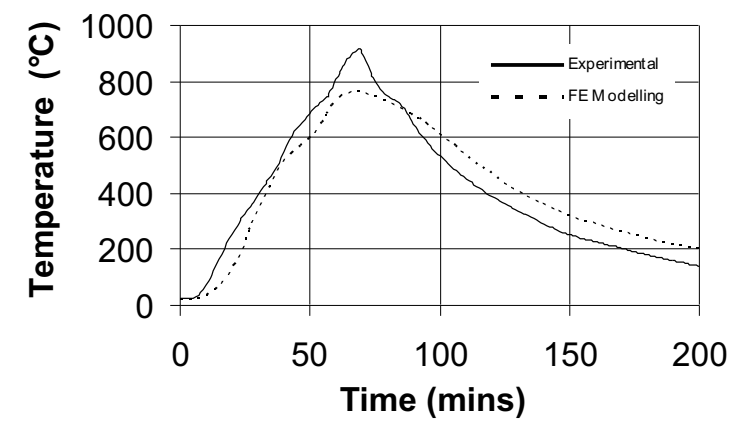

Fig. 5: Finite element for flexible end plate: bottom of connection

Results from the finite element modelling are shown in Figs. 4 and 5 and all show close correlation with experimental data. Fig. 6 shows that the biggest difference between predicted and experimental temperatures is on the underside of the beam upper flange. This is due to the concrete being excluded from the analysis.

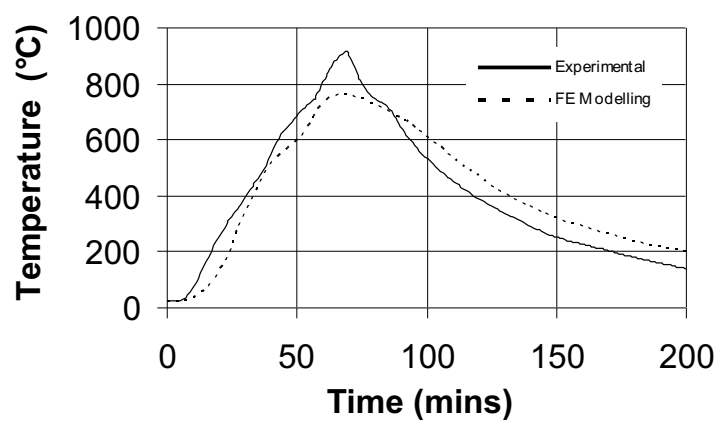

Fig. 6: Finite element for flexible end plate: top flange of beam
For detailed calculations where the exact connection geometry is known, this method provides accurate results. It can be used for all connection types where a detailed knowledge of its response in fire is required. This method, however, is time consuming both in model creation and simulation run time. It could not, therefore, be a day-to-day modelling approach.

\section{Conclusions}

This paper has investigated three methods for predicting connection temperatures. The Eurocode suggests connection temperatures can be calculated as percentages of the mid-span beam flange temperature. However, results show this method to be unreliable, and it should therefore be used with caution.

The lumped capacitance method, based on the heated surface area of the connection and its volume, showed good correlation with average connection temperatures. More work should be done to look at predicting temperatures of individual connection elements and to definite what volume of the connection beams and columns should be included in calculations.

The Abaqus modelling also showed good correlation with experimental results. This method can therefore be recommended if a detailed temperature profile is needed for mechanical analysis. During the modelling it was found that the inclusion of the concrete slab did not affect the predicted temperatures of the connection. Therefore it does not have to be included, allowing for much quicker computational times.

A detailed yet simple method for predicting connection temperatures is still unavailable and therefore more work is required in this field.

\section{Acknowledgment}

The authors wish to thank Arup Fire and EPSRC for funding this research. They also thank Dr. Y. Wang and Dr. X. Dai of the University of Manchester for allowing access to experimental results in advance of publication.

\section{References}

[1] Bailey, C. G., Lennon, T., Moore, D. B.: The Behaviour of Full-Scale Steel Framed Buildings Subjected to Compartment Fires. The Structural Engineer, April 1999, p. 15-21.

[2] CEN, EC 3: Design of Steel Structures part 1.2: General rules - Structural Fire Design, BS EN 1993-1-2:2005, Brussels: CEN, European Committee for Standardisation, 2006b.

[3] ABAQUS User's Manual, version 6.6, Providence, RI, USA.

[4] Incropea, F. P., DeWitt, D. P., Bergman, T. L., Lavine, A. S.: Fundamentals of Heat and Mass Transfer. John Wiley \& Sons, Hoboken, USA, Sixth Edition, 2005.

[5] Dai, X. H., Wang, Y. C., Bailey, C. G.: Temperature Distributions in Unprotected Steel Connections in Fire. Proc. Steel \& Composite Structures, Manchester, UK, 2007, p. 535-540.

[6] Dai, X. H., Wang, Y. C., Bailey, C. G.: Effects of Partial Fire Protection on Temperature Developments in Steel 
Joints Protected by Intumescent Coating. Fire Safety J., Jan. 2009, p. 16-32.

[7] Lennon, T., Moore, D. B.: Results and Observations from Full-Scale Fire Test at BRE Cardington, 16 January 2003. BRE, Watford, UK, 2004.

Kate Anderson

e-mail:s0091706@sms.ed.ac.uk

\section{Martin Gillie}

University of Edinburgh

School of Engineering and Electronics

Edinburgh, United Kingdom 